\title{
An Improved WSN Data Integration Scheme Base on BP Neural Network
}

\author{
Youwei Shao ${ }^{1}$ \\ ${ }^{1}$ School of Applied Electronics, Chongqing College of Electronic Engineering, \\ Chongqing, China \\ E-mail: cqshaoyouwei@126.com
}

\begin{abstract}
In forest fire monitoring, in order to achieve the goal of reducing a large number of invalid and redundant data in wireless sensor network, improving the convergence rate of the wireless sensor network, prolonging the life cycle of nodes, improving the accuracy of fire report, this paper proposed an improved data integration method based on BP neural network. Data generated by various sensors can be integrated on the nodes with this method, the convergence speed of BP neural network can be improved by reference of real-time processing capacity of the node, and thus the energy consumption was reduced to a great extent. The experimental results showed that the proposed method can be well applied in fire monitoring sensor network, the monitoring accuracy was improved and the energy consumption of nodes was reduced, the capacity of wireless sensor network for forest fire monitoring was increased significantly.
\end{abstract}

Keywords: Forest fire monitoring; Wireless sensor network; BP neural network; Data integration; Energy consumption of nodes

\section{Introduction}

To detect the forest environment through different equipment, and transmit the results by using the Internet based on this, the fire monitoring capacity can be largely increased. Using sensor technology to monitor forest fire so as to save nature [1], however, the current technology may generate a large number of invalid and redundant data in wireless sensor network, so data transmission speed of wireless sensor network is greatly reduced, therefore the research of this direction is becoming more and more critical. In order to reduce the cost of nodes, relevant experts and scholars put forward a series of related techniques, such as node scheduling, etc. [2], the life cycle of the nodes were improved to a great extent. By introducing data integration technology in the wireless sensor network, if the fire is detected, using center node to notify data center, so the fire situation is transferred based on this. The node module is in Quiesced State if there is no fire, therefore the energy consumption of nodes is significantly reduced [3-4]. So, it is very necessary to realize data integration in wireless sensor network. But the technology also has shortcomings, mainly includes: there are many data integration algorithms, so to determine the ideal method from these algorithms to detect fires has the certain difficulty; the algorithm with relative smaller convergence rate may be a cause in this aspect, a lot of energy required to be consumed, and so on. According to the mentioned issues above, we clarified the optimized data integration method, this technology has relative higher convergence speed, and illustrated its specific implementation process. Based on this, introduced the technology into forest fire prevention, the results showed that it reduced the invalid data to a great extent, at the same time the lifetime of nodes can also be increased. 


\section{Algorithm Design}

\subsection{Overall Architecture}

Mainly includes the several layers (Figure 1) as follows, add integration estimation layer in general architecture, it cooperates with upper and lower layer to remove the invalid data together, so as to realize the effective integration of multiple data.

For induction layer, using different types of sensors for data acquisition, parameters such as temperature, humidity and so on are mainly involved.

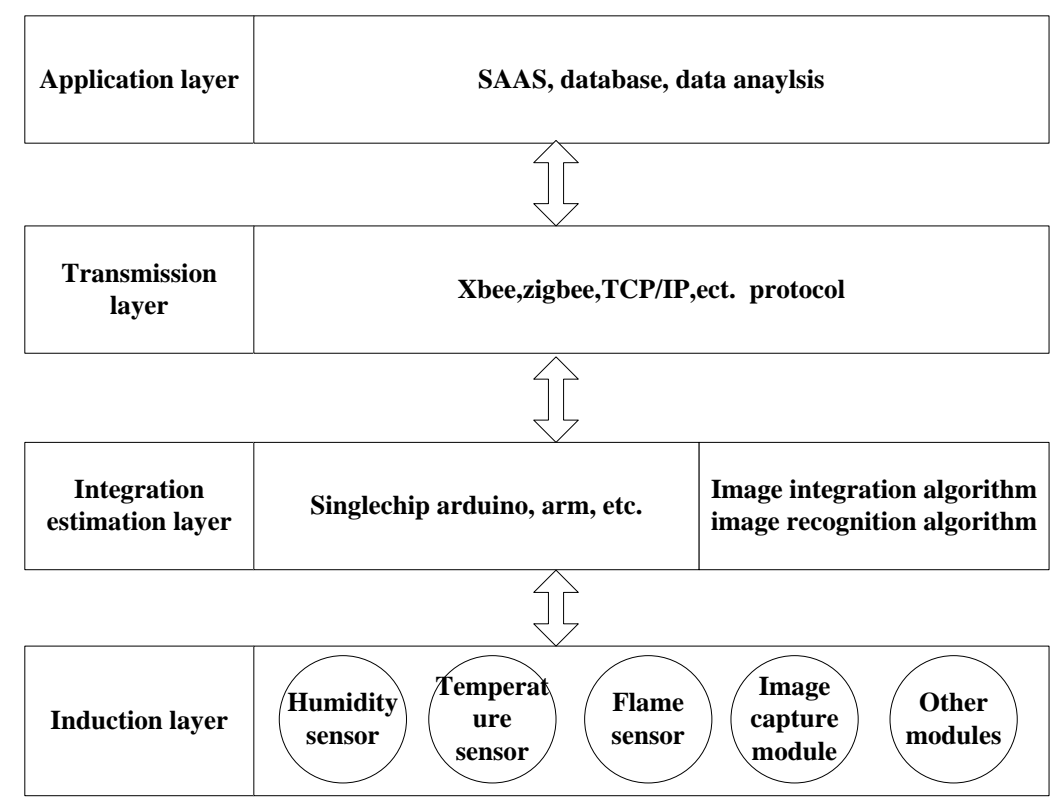

Figure 1. System Network Layer

\subsection{Algorithm Proposed}

Data integration indicates the decision-making process to process information via the computer, control them and make a scientific and rational. First of all, through wireless devices for data acquisition, and then implement information processing and control by single-chip and server, finally to make the whole system to make decisions.

In 1934, Warren $\mathrm{NcCl} / \mathrm{Och}$ and WAl ter Ptts illustrated artificial neuron model in research process for the first time, to be specific, its features including: weighting, threshold of input signals of contrast neurons, and to determine its output by this way. Based on the above theory, we obtain neural network method after adjusting threshold value.

Algorithm optimization: Using Levenberg - Narquardt algorithm for improvement, the convergence time is shortened to a great extent, so it can be effectively applied on sensor with relative less electric quantity.

\subsection{Neural Network Training}

All the nodes are configured with three sensors, which are responsible for the reception of information such as temperature, humidity, gas (UH), etc. successively. The first step is to set threshold; the second step is to get the input vector.

Definition 1 Input vector is the output value $P(X 1, X 2, X 3)$ after three sensors achieved the preset threshold value. For instance, when the analog signals of 
temperature, humidity, gas are $40,60,100$ respectively, in this case, the input vector is $P(40,60,100)$.

It can be seen from Fig. 2 that, when the center node is implemented, the neurons of $M \mathbf{P}$ handles processing of data. First step, $\mathbf{M} P$ reaches the learning phase, properly modify the weights and thresholds. In the case of multi-layer network, the lower the inputs and outputs of upper and lower layer are equal, the output results are regarded as the reference for the fire determination. To train neural network training by using the function of $N$ At Lab, and modify the weights and threshold values repeatedly on this basis, center node transfers to terminal node, thus the latter one can obtain the capacity of fire interpretation, without transporting many data, and reduce the energy consumption obviously.

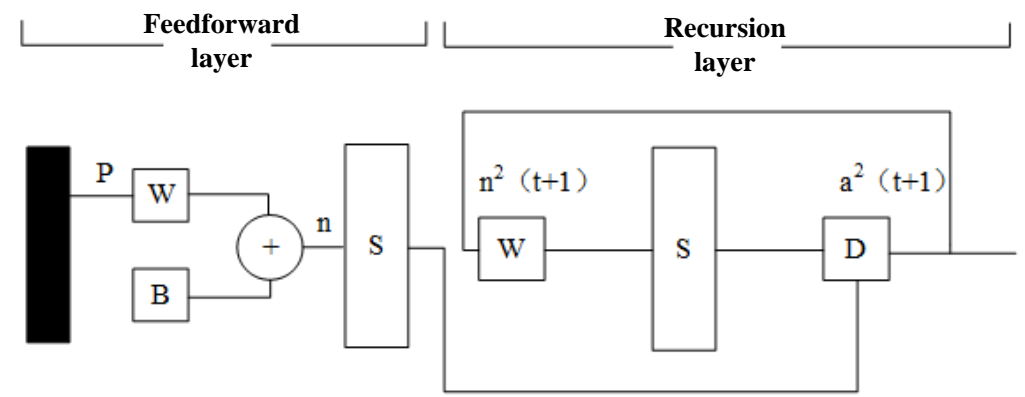

Figure 2. Neurons

If the singlechip applied in nodes cannot guarantee its effective training on sensor completely, in this case, there are mainly four steps to complete learning phase on data center as follows:

(1) Using center node CenterNode, CN)to reach learning phase, the latter one set the weights and threshold values on network neurons by using $Z$ gbee network.

(2) $\mathrm{CN}$ chooses collected data of the area within some time range, and build neural network on this basis.

(3) $\mathrm{CN}$ searches in Database, and structure the input vector $P(X 1, X 2, X 3)$, based on this, training on $P$, and obtain new weights and threshold values at the end.

\subsection{CN Transports the Obtained Results in step 3 to Terminal Node}

2.4.1. Estimation Node vs. Input: After the threshold values and weights are obtained, terminal node can estimate fire condition by using neurons. To calculate output vector by using input vector, the specific procedures are as follows:

(1) The input vector multiplied by a random formed weight matrix. Plus threshold value $B$ on this basis, and the net input $n$ is structured, $n=W_{1} P_{1}+W_{.2} P_{2}+\cdots W_{. R} P_{R}+B$.

(2) Output the neurons of feedforward layer. Using transmission function to deal with net input, the feedforward neural output can be obtained by this way, and regard it as input $P=$ Purel $i(n)$ ).

(3) To solve recursive layer repeatedly up to convergence Art put $_{1}=$ Posl i n $\left(W_{i-1}+B\right)$.

2.4.2. Convergence Procedure Optimization: The effect of improved $B P$ neural network by Leveberg - NArquardt algorithm (LM algorithm) is very good, and 
energy consumption has fallen dramatically. Its convergence has improved, so its data transmission time is reduced then.

Leveberg - NArquardt algorithm belongs to the scope of Quasi-Newton algorithm, its specific procedures include:

(1) Set initial weight value vector $r_{0}$ randomly, and set objective error $\varepsilon, \quad k:=1$

(2) Assumed that $[-1,1]$ with continuous function $\phi(\mathrm{x})$, and select $m$ training samples from it, and preset equation set:

$$
\left.\begin{array}{l}
f_{i}(\mathrm{r})=\sum_{i=1}^{n} r_{i} \quad T_{i}\left(\mathrm{x}_{j}\right)-\phi^{\wedge}\left(\mathrm{x}_{j}\right), \\
\mathrm{i}=1,2,3, \cdots, \mathrm{m} ; \mathrm{j}=1,2,3, \cdots, \mathrm{m}
\end{array}\right\}
$$

In above formula, ${ }^{r_{i}}$ denotes the total number of training samples.

To define $F(\mathrm{r})$, the specific definition as shown below:

$$
\begin{aligned}
& F(r):=\left[f_{1}(r) f_{2}(r) \cdots f_{m}(r)\right] \\
& E(\mathrm{x})=\sum_{i=1}^{n}\left(\phi\left(\mathrm{x}_{j}\right)-\phi^{\wedge}\left(\mathrm{x}_{j}\right)\right)^{2}
\end{aligned}
$$

Here, $\phi^{\wedge}(\mathrm{x})$ 表示 denotes the unknown mapping relation to be solved, in general, only can get part of its samples. The purpose of network training is to correct the $r$ vector of output layer, and the $E$ optimization can be realized by using this method, therefore, the optimal approximation relation exists between $\phi^{\wedge}\left(\mathrm{x}_{j}\right)$ and $\phi\left(\mathrm{x}_{j}\right)$.

To solve Jacobi matrix $J$ of $r$ and formula (1) .

(3) The following formula is given.

$$
\begin{aligned}
R^{(\mathrm{k}+1)} & =r^{(\mathrm{k})}-\eta_{k}\left(\mathrm{~J}^{T}\left(\mathrm{R}^{(\mathrm{k})}\right) \mathrm{J}\left(\mathrm{r}^{(\mathrm{k})}\right)\right. \\
& \left.+\mu_{k} \mathrm{I}\right) \mathrm{J}^{T}\left(\mathrm{r}^{(\mathrm{k})}\right) \mathrm{F}\left(\mathrm{r}^{(\mathrm{k})}\right)
\end{aligned}
$$

And then search on it.

(4) Substitute this formula to $E$, the condition shall be satisfied here: $E\left(\mathrm{r}^{(\mathrm{k})}\right) \leq \varepsilon$, In this case, to terminate algorithm and obtain the standard of weight vector, otherwise, continue the subsequent processes.

(5) If $E\left(\mathrm{r}^{(\mathrm{k}+1)}\right)-\mathrm{E}\left(\mathrm{r}^{(\mathrm{k})}\right) \leq 0$, in such case, let $\mu_{k}=\mu_{k} / 4$; otherwise $\mu_{k}=\mu_{k \times} 4$, and proceed with 3$)$.

Here, the definition of $\mathrm{J}$ is as follows:

$$
J\left(\mathrm{r}^{(\mathrm{k})}\right):\left[\begin{array}{cccc}
\frac{\partial f_{1}\left(\mathrm{r}^{(\mathrm{k})}\right)}{\partial r_{1}} & \frac{\partial f_{1}\left(\mathrm{r}^{(\mathrm{k})}\right)}{\partial r_{2}} & \cdots & \frac{\partial f_{1}\left(\mathrm{r}^{(\mathrm{k})}\right)}{\partial r_{n}} \\
\frac{\partial f_{2}\left(\mathrm{r}^{(\mathrm{k})}\right)}{\partial \omega_{1}} & \frac{\partial f_{2}\left(\mathrm{r}^{(\mathrm{k})}\right)}{\partial \omega_{2}} & \cdots & \frac{\partial f_{2}\left(\mathrm{r}^{(\mathrm{k})}\right)}{\partial \omega_{n}} \\
\vdots & \vdots & & \vdots \\
\frac{\partial f_{m}\left(\mathrm{r}^{(\mathrm{k})}\right)}{\partial r_{1}} & \frac{\partial f_{m}\left(\mathrm{r}^{(\mathrm{k})}\right)}{\partial r_{2}} & \ldots & \frac{\partial f_{m}\left(\mathrm{r}^{(\mathrm{k})}\right)}{\partial r_{n}}
\end{array}\right]
$$

Where, weights and bias value increment can be solved by the following formula, specific steps as follows:

$$
\square x(\mathrm{k})=-\left[J^{T}\left(\mathrm{x}_{k}\right) \mathrm{J}\left(\mathrm{x}_{k}(\mathrm{k})\right)+\mathrm{ul}\right]^{-1} J^{T}\left(\mathrm{x}_{k}\right) \mathrm{F}\left(\mathrm{x}_{k}\right)
$$




$$
H\left(\mathrm{x}_{k}\right)=\mathrm{J}^{T}\left(\mathrm{x}_{k}\right) \mathrm{J}\left(\mathrm{x}_{k}\right), \mathrm{G}=\mathrm{H}+\mathrm{ul}
$$

In above formula, $J$ represents Jacobi matrix, through simplifying processing to obtain the following formula:

$$
\square X=-G^{-1} J^{T} F
$$

Considering the shortcoming of relatively more time required by large matrix inversion, combined with the feature of symmetric positive definite of matrix at the same time, which illuminates the convergence of the algorithm can be improved. Formula (3) involved inversion process of large matrix $G$, under the condition of the optimal algorithm, its time complexity still was $O\left(\mathrm{n}^{3}\right)$, of which $G^{-1} J F$ involved the same matrix multiplication, the causes mentioned above led to large time-consuming of BP algorithm.

To change the form of formula (3), as follows

$$
G\left(\mathrm{x}_{k}\right) \square \mathrm{x}_{k}=-J^{T}\left(\mathrm{x}_{k}\right) \mathrm{F}\left(\mathrm{x}_{k}\right)
$$

After deformation, one can calculate the weight value increment $\square X$ by using linear system of equations, to deal with $\square X$ with $L U$ decomposition method, and $n^{3} / 3$ operations are involved, thus the time complexity is reduced significantly. Even for simple networks, set $n$ as 100 , this algorithm can reduce at least 60 ten thousands operations.

\section{Experiment}

\subsection{Implementation of the Center Node}

For CN, it mainly involves the following modules (see Figure 3), we will explain each module separately.

Sensing module: use a variety of sensors for data acquisition work and provide conditions for subsequent data integration.

Communication module: hardware equipment to be able to play a role is Xbee protocol stack.

Processing module: using microcontroller for data integration and calculation, to determine the effective information with this method, based on this to transport it to a wireless module, and it also has the function of power management, to provide power strategy for the system.

Data acquisition module: its main function is to drive the sensors for data acquisition, using the threshold to remove the invalid information directly, and eventually achieve the goal of reducing power consumption.

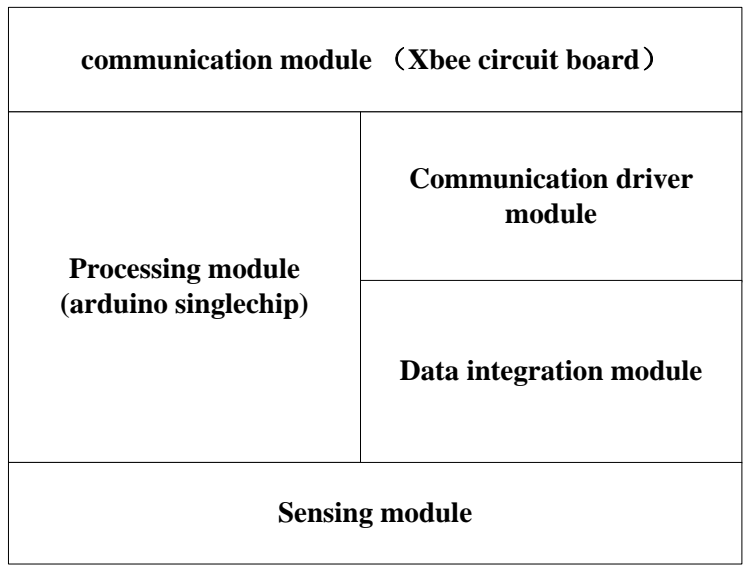

Figure 3. Center Nodes 
Data integration module: this part is mainly responsible for the information integration of different types, to form integrated information, and report the fire tendency. In the process of integration, that is the method we illustrated above, to integrate different types of data through the neural network, and then improve the convergence speed through Leveberg - NAr quar dt algorithm.

$\mathrm{CN}$ is a special device. The modularization of system can make nodes to be equipped be with different types of sensors in accordance with the environmental conditions for the integration of various kinds of data. In node sleep mode, only open the acquisition and integration module. When the latter module detected the fire nodes are activated, and it can open communication module. The introduction of this system into forest fire prevention network, can obviously reduce the power consumption of the node.

Problem worth especial attention is that, the algorithm designed in this paper used by $\mathrm{CN}$ can largely improve the convergence speed, and the requirements on hardware is reduced somewhat. Through ordinary embedded microcontroller can make integration algorithm realized. And the status of $B P$ neural network algorithm unable to be transplanted to the node is solved ultimately.

\subsection{Experimental Procedure}

(1) To debug $Z$ gBee module, construct network. In specific, it mainly debug $Z$ gBee module through $X$ bee adaptor to $A P$ mode;

(2) To develop CN, insert $Z$ gBee into the expansion board of Ardui no, through the driven of I D E designed $Z$ gBee of Ardui no, based on this, insert it into singechip;

(3) To realize neural network by $N$ At Lab on computer, based on this, it transfers into $C$ program, then transplants it to $\mathrm{CN}$.

(4) to obtain new threshold and weight after the data integration complete on $\mathrm{CN}$.

(5) $\mathrm{CN}$ transports threshold and weight to terminal nodes through network.

(6) To develop the terminal nodes, equipped with sensors, and then implement the sampling work, to complete data integration with threshold and weight provided by $\mathrm{CN}$. Based on this unsing microcontroller programming to transfer the effective formed data to network center;

(7) To monitor the receive information.

\section{Experimental Results}

The sample data selected in this paper was training network based on DRNN model, input was $u(\mathrm{k})$, and output was $y(\mathrm{k})$, for diagonal recurrent neural network was a three-layer network [16]. The selected model structure was $(2,7,1)$, the system simulation test was carried on with traditional BP algorithm and improved LM_BPI algorithm as comparison, test for 50 times, and calculated the average, each algorithm scheduled training steps and error requirements, and simulation accuracy comparison results as shown in table 1 and table 2 .

Table 1. Simulation Preset Parameters and Comparison Result 1

\begin{tabular}{cccc}
\hline learning algorithm & Preliminary training steps & average time/s & average error \\
\hline LM_BPI & 5000 & 50.1 & 0.0074 \\
BP & 5000 & 20.2 & 0.0193 \\
\hline
\end{tabular}


Table 2. Simulation Preset Parameters and Comparison Result 2

\begin{tabular}{cccc}
\hline learning algorithm & Preliminary training steps & error & average time/s \\
\hline LM_BPI & 5253 & $<=0.001$ & 23.12 \\
BP & 10138 & $<=0.001$ & 107.58 \\
\hline
\end{tabular}

This paper, by introducing LM_BPI algorithm into DRNN weight training, to avoid the defect of local convergence and slow convergence speed produced by traditional BP algorithm, the simulation experiment included several common attack types, random sampling data in the intrusion detection database, in order to make sure the correctness of the experiment. Simulation comparison 1, training steps of the BP algorithm and the optimized LM_BPI algorithm were set to be 5, 000, by comparison, with the same training steps, time needed for BP algorithm was about 1.5 times that of the time required for the optimized LM_BPI algorithm.

Simulation comparison 2, the BP algorithm training steps were set to 10000 , and practical training steps was 10138, as shown in Figure 4, through the comparison with the simulation experiment 1 , the increase of the training steps, time needed for BP algorithm was proportional to the training steps, so autonomous learning was poor. The optimized LM_BPI algorithm training steps were 5253 times, by comparison, the stability of LM_BPI algorithm was superior to BP algorithm, consistent with the results of simulation comparison 1. Compared with ordinary node, using data integration, the life cycle of nodes was obviously improved, along with the increase of the number of nodes, the information transmission on several nodes consumed relatively higher power, the advantages of the latter one was promoted constantly. In Figure 4, it made power consumption status comparison of the above two kinds of nodes, Figure 5 showed the convergence speed of the introduced algorithm in this paper. In the following experiment, we used ordinary nodes, conventional integration nodes, $B P$ neural network integration nodes as the multiterm, compared the differences among the three. As we can see in Figure 4, along with the increase of the number of nodes, energy consumption of a single node was also increasing. It can be known from comparison study that, the power consumption of the third node was a third of the first one, at the same time accompanied by the increase of the number of nodes, and the increasing ratio of power consumption was relatively small. Therefore, the survival time of nodes after our designed method ( $B P$ )integration was also increased.

The convergence rate was compared at the end of the experiment, when the rate of $C P U$ was more than $2000 h z$, convergence speed was only associated with the node number. As a result, the algorithm had very good effect. Through the analysis of the output we can observe that, after optimizing, the integration algorithm improved the prediction success rate to a great extent, but also made the energy consumption reduced. Integration with the $Z g B e e$ type network of $V B N$, the goal of the long distance and low power trace message transfer was achieved. Problems of W $\$ N$ existing in hardware were effectively solved. 


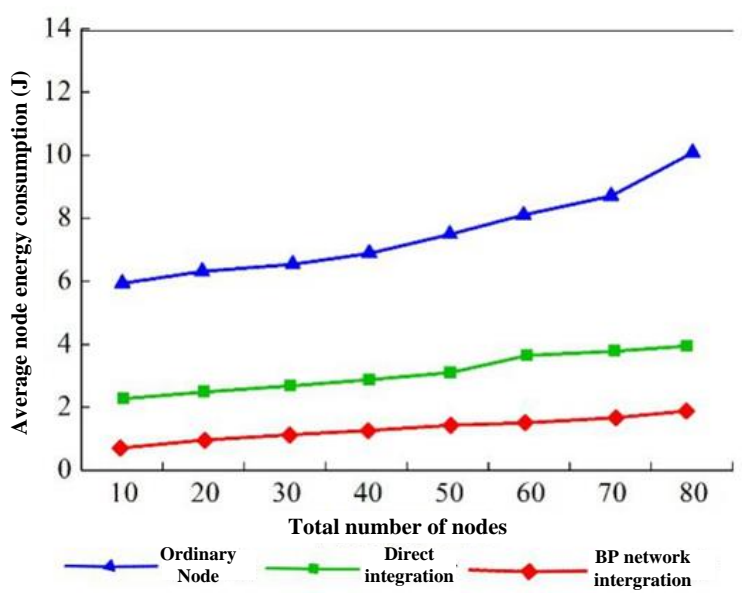

Figure 4. Energy Consumption

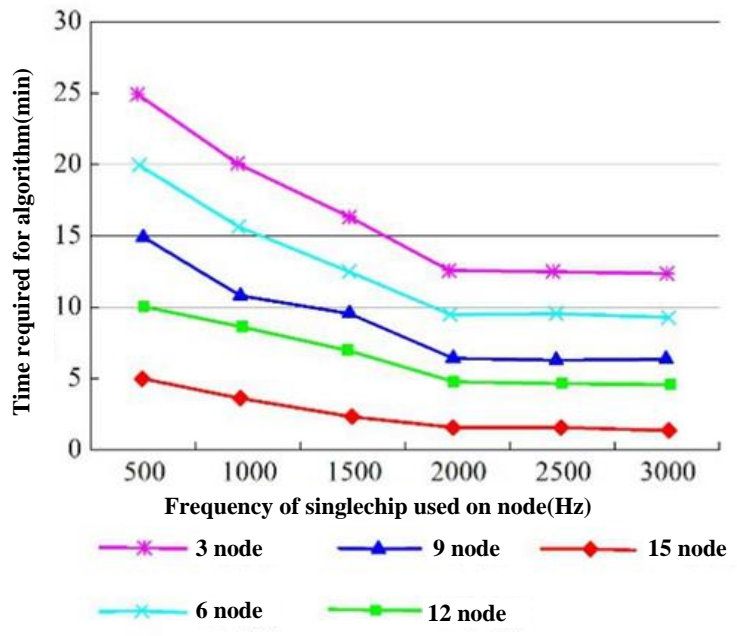

Figure 5. Algorithm Time

\section{Conclusion}

The proposed detection scheme using threshold remove the useless information in the integration layer of sensing system, through the relevant method to process the data collected in induction layer. And then to sort by integration algorithm according to the time order. Then using the integration method of $B P$ neural network, and improve the method with Levenberg - Narquar dt algorithm. The experimental results showed that this method to a great extent, shortened the convergence time, made it effectively applied on sensor with relative less power, which can largely improve the accuracy of prediction, and can also improve the efficiency of data monitoring, effectively reduce energy consumption.

\section{Acknowledgement}

The research is supported by Chongqing Municipal Education Commission science and technology research project (KJ132202). 


\section{References}

[1] D. Jiang, Z. Xu and Z. Lv, "A multicast delivery approach with minimum energy consumption for wireless multi-hop networks", Telecommunication Systems, (2015), pp. 1-12.

[2] Y. Geng, J. Chen, R. Fu, G. Bao and K. Pahlavan, "Enlighten Wearable Physiological Monitoring systems: On-Body RF Characteristics Based Human Motion Classification Using a Support Vector Machine", no. 99, (2015), pp. 1-16.

[3] X. Song and Y. Geng, "Distributed Community Detection Optimization Algorithm for Complex Networks", Journal of Networks, vol. 9, no. 10, (2014), pp. 2758-2765.

[4] K. Pahlavan, P. Krishnamurthy and Y. Geng, "Localization Challenges for the Emergence of the Smart World, Access, IEEE, vol. 3, no. 1, (2015), pp. 1-11.

[5] J. He, Y. Geng, Y. Wan, S. Li and K. Pahlavan, "A cyber physical test-bed for virtualization of RF access environment for body sensor network", Sensors Journal, IEEE, vol. 13, no. 10, (2013), pp. 3826-3836.

[6] Z. Lv, A. Tek and F. Da Silva, "Game on, science-how video game technology may help biologists tackle visualization challenges", PloS one, vol. 8, no. 3, (2013), 57990.

[7] T. Su, W. Wang and Z. Lv, "Rapid Delaunay triangulation for randomly distributed point cloud data using adaptive Hilbert curve", Computers \& Graphics, vol. 54, (2016), pp. 65-74.

[8] J. Hu, Z. Gao and W. Pan, "Multiangle Social Network Recommendation Algorithms and Similarity Network Evaluation", Journal of Applied Mathematics, 2013 (2013).

[9] S. Zhou, L. Mi, H. Chen and Y. Geng, "Building detection in Digital surface model", 2013 IEEE International Conference on Imaging Systems and Techniques (IST), (2012).

[10] J. He, Y. Geng and K. Pahlavan, "Toward Accurate Human Tracking: Modeling Time-of-Arrival for Wireless Wearable Sensors in Multipath Environment”, IEEE Sensor Journal, vol.14, no. 11, (2014), pp. 3996-4006.

[11] Y. Lin, J. Yang and Z. Lv, "A self-assessment stereo capture model applicable to the internet of things", Sensors, vol. 15, no. 8, (2015), pp. 20925-20944.

[12] Y. Liang*, "Satisfaction with Economic and Social Rights and Quality of Life in a Post-Disaster Zone in China: Evidence from Earthquake-Prone Sichuan", Disaster Medicine and Public Health Preparedness, vol. 9, no. 2, pp. 111-118.

[13] Y. Liang*, "Correlations Between Health-Related Quality of Life and Interpersonal Trust: Comparisons Between Two Generations of Chinese Rural-to-Urban Migrants", Social Indicators Research, vol.123, no. 3, pp. 677-700.

[14] Y. Liang* and P. Lu, "Medical insurance policy organized by Chinese government and the health inequity of the elderly: longitudinal comparison based on effect of New Cooperative Medical Scheme on health of rural elderly in 22 provinces and cities", International Journal for Equity in Health, vol. 13 , no. 37, (2014), pp. 1-15, DOI:10.1186/1475 -9276-13-37.

[15] Y. Liang* and D.i Zhu, "Subjective Well-Being of Chinese Landless Peasants in Relatively Developed Regions: Measurement Using PANAS and SWLS", Social Indicators Research, vol. 123, no. 3, pp. 817-835.

[16] Y.Liang* and X. Wang, "Developing a new perspective to study the health of survivors of Sichuan earthquakes in China: a study on the effect of post-earthquake rescue policies on survivors' healthrelated quality of life", Health Research Policy and Systems, vol. 11, no. 41, pp. 1-12, DOI:10.1186/1478-4505-11-4

[17] J. Hu and Z. Gao, "Modules identification in gene positive networks of hepatocellular carcinoma using Pearson agglomerative method and Pearson cohesion coupling modularity", Journal of Applied Mathematics, 2012 (2012).

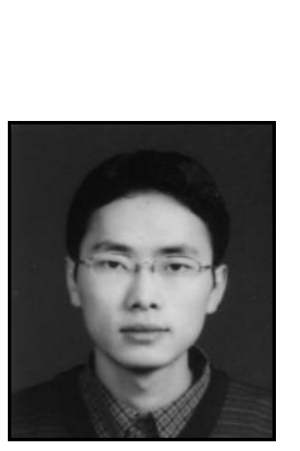

\section{Author}

Shao Youwei, he is an associate professor at Chongqing College of Electronic Engineering. Born in 1979, Mr. Shao received master degree from Chongqing University, and his main research interests are Sensing and Control. He has published several research papers in scholarly journals in the above research areas. 
International Journal of Future Generation Communication and Networking Vol. 9, No.9, (2016) 